\title{
ASIENKUNDLICHE PUBLIKATIONEN DER ÖSTERREICHISCHEN AKADEMIE DER WISSENSCHAFTEN
}

DsÖAW Denkschriften der philosophisch-historischen Klasse der Österreichischen Akademie der Wissenschaften

SbÖAW Sitzungsberichte der philosophisch-historischen Klasse der Österreichischen Akademie der Wissenschaften

Veröffentlichungen zu den Sprachen und Kulturen Südasiens

Ehemals: Veröffentlichungen der Kommission für Sprachen und Kulturen Südasiens

1 Tilmann Vetter, Erkenntnisprobleme bei Dharmakīti. [SbÖAW 245/2]. Wien 1964. 118 p. (vergriffen)

2 Lambert Schmithausen, Mandanamiśra's Vibhramavivekah. Mit einer Studie zur Entwicklung der indischen Irrtumslehre. [SbÖAW 274/1]. Wien 1965. 270p. (€ 37.-)

3 Tilmann Vetter, Dharmakīrti's Pramānaviniśsayah. 1. Kapitel: Pratyaksam. Einleitung, Text der tibetischen Übersetzung, Sanskritfragmente, deutsche Übersetzung. [SbÖAW 250/3]. Wien 1966. 112p. (vergriffen)

4 Ernst Steinkellner, Dharmakīti's Hetubinduh. Teil I: Tibetischer Text und rekonstruierter Sanskrit-Text. [SbÖAW 252/1]. Wien 1967. 116p. (vergriffen)

5 Ernst Steinkellner, Dharmakīrti's Hetubinduh. Teil II: Übersetzung und Anmerkungen. [SbÖAW 252/2]. Wien 1967. 220p. (vergriffen)

6 Erich Fraunallner, Materialien zur ältesten Erkenntnislehre der Karmamūmāmsāa [SbÖAW 259/2]. Wien 1968. 114p. (€ 20.-)

7 Tilmann Vetter, Mandanamiśra's Brahmasiddhih. Brahmakāndah. Übersetzung, Einleitung und Anmerkungen. [SbÖAW 262/2]. Wien 1969. 126p. (€ 23.20)

8 Lambert Schmithausen, Der Nirvāna-Abschnitt in der Viniśsayasamgrahañ̄ der Yogācārabhümih. [SbÖAW 264/2]. Wien 1969. 216p. mit 2 Tabellen (vergriffen)

9 Erich Frauwallner, Die Lehre von den zusätzlichen Bestimmungen (upādhih) in Gañgeśa's Tattvacintāmaṇih. [SbÖAW 266/2]. Wien 1970. 72p. (vergriffen)

10 Gerhard Oberhammer, Yāmunamuni's Interpretation von Brahmasūtram 2,2,42-45 Eine Untersuchung zur Pāñcarātra-Tradition der Rāmānuja-Schule. [SbÖAW 274/4]. Wien 1971. 136p. (€ 13.50)

11 Tilmann Vetter, Sarvajñātman's Saṃssepaśarīrakam. 1. Kapitel. Einführung, Übersetzung, Anmerkungen. [SbÖAW 282/3]. Wien 1972. 176p. (vergriffen)

12 Ernst Steinkellner, Dharmakīrti's Pramānaviniścayah. 2. Kapitel: Svārthānumānam. Teil I: Tibetischer Text und Sanskrittexte. [SbÖAW 287/4]. Wien 1973. 120p. (€ 17.40)

13 Gerhard Oberhammer, Strukturen yogischer Meditation. Untersuchungen zur Spiritualität des Yoga. [SbÖAW 322]. Wien 1977. 244p. (vergriffen)

14 Gerhard Oberhammer, Materialien zur Geschichte der Rāmānuja-Schule I. Parāśarabhattas Tattvaratnākarah. [SbÖAW 346]. Wien 1979. 258p. (vergriffen) 
15 Ernst Steinkellner, Dharmakīrti's Pramānaviniścayah. 2. Kapitel: Svārthānumānam. Teil II: Übersetzung und Anmerkungen. [SbÖAW 358]. Wien 1979. 164p. (€ 25.40)

16 Hertha Krick $(\dagger)$, Das Ritual der Feuergründung (Agnyādheya). Hrsg. Gerhard Oberhammer. [SbÖAW 399]. Wien 1982. 682p. (€ 49.40)

17 Gopikamohan Bhattacharya, Yajñapati Upādhyāya's Tattvacintāmaniprabhā (Anumānakhandah). [SbÖAW 423]. Wien 1984. 198p. (€ 20.30)

18 Gerhard Oberhammer, Wahrheit und Transzendenz. Ein Beitrag zur Spiritualität des Nyāya. [SbÖAW 424]. Wien 1984. 256p., 1 Farbtafel. (€ 30.50)

19 Erich Frauwallner, Nachgelassene Werke I. Aufsätze, Beiträge, Skizzen. Hrsg. Ernst Steinkellner. [SbÖAW 438]. Wien 1984. 144p. (€ 25.30)

20 Oskar von Hinüber, Das ältere Mittelindisch im Überblick. [SbÖAW 467]. 2., erweiterte Auflage. Wien 2001. 344p. (€ 45.10)

21 Roque Mesquita, Yāmunācāryas Samvitsiddhi. Kritische Edition, Übersetzung und Anmerkungen mit einem Rekonstruktionsversuch der verlorenen Abschnitte. [SbÖAW 504]. Wien 1988. 200p. (€ 25.50)

22 Max Sparreboom - Johannes C. Heesterman, The Ritual of Setting Up the Sacrificial Fires according to the Vādhūla School (Vādhülaśrautasūtra 1.1-1.4). [SbÖAW 539]. Wien 1989. 146p. (vergriffen)

23 Walter Slaje, Katalog der Sanskrit-Handschriften der Österreichischen Nationalbibliothek (Sammlungen Marcus Aurel Stein und Carl Alexander von Hügel). [SbÖAW 546]. Wien 1990. 152p., 2 Farb- und $5 \mathrm{~S} / \mathrm{W}$-Tafeln. (€ 20.30)

24 Roque Mesquita, Yāmunācāryas Philosophie der Erkenntnis. Eine Studie zu seiner Saṃvitsiddhi. [SbÖAW 563]. Wien 1990. 314p. (€ 35.60)

25 Michael T. Much, Dharmakīrtis Vādanyāya. Teil I: Sanskrit-Text. XXX, 75p. Teil II: Übersetzung und Anmerkungen. [SbÖAW 581]. Wien 1991. XXI, 135p. (€50.80)

26 Erich Frauwallner, Nachgelassene Werke II. Philosophische Texte des Hinduismus. Hrsg. Gerhard Oberhammer - Chlodwig H. Werba. [SbÖAW 588]. Wien 21998. 301p. $(€ 30.50)$

27 Walter Staje, Vom Mokșopāyaśāstram zum Yogavāsișthamahārāmāyaṇa. Philologische Untersuchungen zur Entwicklungs- und Überlieferungsgeschichte eines indischen Lehrwerks mit Anspruch auf Heilsrelevanz. [SbÖAW 609]. Wien 1994. 338p. $(€ 62.80)$

28 Gerhard Oberhammer, Materialien zur Geschichte der Rāmānuja-Schule II. Vātsya Varadagurus Traktat von der Transzendenz des Brahma in der kontroverstheologischen Tradition der Schule. [SbÖAW 633]. Wien 1996. 115p. (€ 22.70)

29 Gerhard Oberhammer, Materialien zur Geschichte der Rāmānuja-Schule III. Yādavaprakāśa, der vergessene Lehrer Rāmānujas. [SbÖAW 646]. Wien 1997. 181p. (€ 32.70)

30 Lambert Schmithausen, Maitri and Magic: Aspects of the Buddhist Attitude toward the Dangerous in Nature. [SbÖAW 652]. Wien 1997. 76p. (vergriffen)

31 Gerhard Oberhammer, Materialien zur Geschichte der Rāmānuja-Schule IV. Der "Innere Lenker" (antaryāmī). Geschichte eines Theologems. [SbÖAW 659]. Wien 1998. 128p. (€ 24.20)

32 Tom J. F. Tillemans, Dharmakīrti's Pramānavārttika. An Annotated Translation of the Fourth Chapter (parārthānumāna). Vol. 1 (k. 1-148). [SbÖAW 675]. Wien 2000. 256p. $(€ 43 .-)$ 
33 Tilmann Vetter, The "Khandha Passages" in the Vinayapitaka and in the Four Main Nikāyas. [SbÖAW 682]. Wien 2000. 357p. (€ 56.20)

34 Gerhard Oberhammer, Materialien zur Geschichte der Rāmānuja-Schule V. Zur Lehre von der ewigen vibhūti Gottes. [SbÖAW 684]. Wien 2000. 154p. (€ 28.20)

35 Gerhard Oberhammer, Materialien zur Geschichte der Rāmānuja-Schule VI. Die Lehre von der Göttin vor Vè̇katanātha. [SbÖAW 696]. Wien 2002. 141p. (€ 28.80)

36 Gerhard Oberhammer, Materialien zur Geschichte der Rāmānuja-Schule VII. Zur spirituellen Praxis des Zufluchtnehmens bei Gott (śaraṇāgati) vor Venkathanātha. [SbÖAW 710]. Wien 2004. 186p. (€ 38.50)

37 Heinz Bechert, Eine regionale hochsprachliche Tradition in Südasien: Sanskrit-Literatur bei den buddhistischen Singhalesen. [SbÖAW 718]. Wien 2005. 173p. (€ 29.90)

38 Gerhard Oberhammer, Materialien zur Geschichte der Rāmānuja-Schule VIII. Zur Eschatologie der Rāmānuja-Schule vor Vè̉kațanātha. [SbÖAW 740]. Wien 2005. 174p. $(€ 44 .-)$

39 Gerhard Oberhammer, Materialien zur Geschichte der Rāmānuja-Schule IX. Der Ātmā als Subjekt in der Theologie Rāmānujas. [SbÖAW 778]. Wien 2008. 161p. (€ 22.-)

\section{Veröffentlichungen zur Sozialanthropologie}

1 Pasang Wangdu - Hildegard Diemberger in cooperation with Guntram Hazod, Shel Dkar Chos 'Byung. Ngag Dbang Skal Ldan Rgya Mtsho. History of the "White Crystal". Religion and Politics of Southern La stod. Translation and Facsimile Edition of the Tibetan Text. [DsÖAW 252]. Wien 1996. 246p. (€ 31.60)

2 Anne-Marie Blondeau - Ernst Steinkellner (ed.), Reflections of the Mountain. Essays on the History and Social Meaning of the Mountain Cult in Tibet and the Himalaya. [DsÖAW 254]. Wien 1996. 273p. (€ 32.70)

3 Anne-Marie Blondeau (ed.), Tibetan Mountain Deities, Their Cults and Representations. Papers Presented at a Panel of the 7th Seminar of the International Association for Tibetan Studies, Graz 1995 (PIATS VI). [DsÖAW 266]. Wien 1998. 214p. (€ 30.50)

4 Niels Gutschow - Axel Michaels - Charles Ramble - Ernst Steinkeldner (ed.), Sacred Landscape of the Himalaya. Proceedings of an International Conference at Heidelberg 22-27 May, 1998. [DsÖAW 308]. Wien 2003. 212p. (€ 64.-)

5 Gebhard Fartacek, Pilgerstätten in der syrischen Peripherie. Eine ethnologische Studie zur kognitiven Konstruktion sakraler Plätze und deren Praxisrelevanz. [SbÖAW 700]. Wien 2003. 219p., 65 Farbabb. (€ 55.-)

6 Johanna Riggler (ed.), Kulturelle Dynamik der Globalisierung. Ost- und westeuropäische Transformationsprozesse aus sozialanthropologischer Perspektive. [SbÖAW 728]. Wien 2005. 305p. (€ 66.-)

7 Johann Heiss (ed.), Veränderung und Stabilität. Normen und Werte in islamischen Gesellschaften. [SbÖOW 729]. Wien 2005. 263p. (€ 65.-)

8 Walter Dostal (ed.), Tribale Gesellschaften der südwestlichen Regionen des Königreiches Saudi Arabien. Sozialanthropologische Untersuchungen. [SbÖAW 732]. Wien 2006. 719p. (€ 80.-) 
9 Andre Gingrich - Guntram Hazod (ed.), Der Rand und die Mitte. Beiträge zur Sozialanthropologie und Kulturgeschichte Tibets und des Himalaya. [SbÖ̈AW 753]. Wien 2006. VI + 301p. (€ 46.40)

10 Per K. Sørensen - Guntram Hazod - Tsering Gyalbo, Rulers on the Celestial Plain. Ecclesiastic and Secular Hegemony in Medieval Tibet. A Study of Tshal Gung-thang. [DsÖAW 361]. Wien 2008. 1001p. (€ 220.80)

11 Martin Slama (ed.), Konflikte-Mächte-Identitäten. Beiträge zur Sozialanthropologie Südostasiens. [SbÖAW 785]. Wien 2009. 324p. (€ 36.-)

12 Brandon Dotson, The Old Tibetan Annals. An Annotated Translation of Tibet's First History. With an Annotated Cartographical Documentation by Guntram Hazod. [DsÖAW 381]. Wien 2009. VI + 319p. (€ 79.20)

Beiträge zur Kultur- und Geistesgeschichte Asiens

1 Ernst Steinkellner, Nachweis der Wiedergeburt. Prajñāsenas 'Jig rten pha rol sgrub pa. Ein früher tibetischer Traktat aus Dunhuang. Mit seinen Glossen diplomatisch herausgegeben, übersetzt und mit Anmerkungen versehen. [Ds ÖAW 197]. Wien 1988. Teil 1: Texte. 46p., 7 Falttafeln. Teil 2: Übersetzung. 54p. (vergriffen)

2 Ernst Steinkellner - Helmut Krasser, Dharmottaras Exkurs zur Definition gültiger Erkenntnis im Pramānaviniścaya (Materialien zur Definition gültiger Erkenntnis in der Tradition Dharmakīrtis 1). Tibetischer Text, Sanskritmaterialien und Übersetzung. [SbÖAW 528]. Wien 1989. 104p. (€ 15.20)

3 Otto Ladstätter - Sepp Linhart (ed.), August Pfizmaier (1808-1887) und seine Bedeutung für die Ostasienwissenschaften. [SbÖAW 562]. Wien 1990. 327p. (€ 35.60)

4 Sylvia Stark, Vātsya Varadagurus Tattvanirnaya. Teil 1: Kritische Textedition. 135p. Teil 2: ̈̈bersetzung und Anmerkungen. 151p. [SbÖAW 570]. Wien 1990. (€ 50.80)

5 János Szerb †, Bu ston's History of Buddhism in Tibet. Critically Edited with a Comprehensive Index. [SbÖAW 569]. Wien 1990. 214p. (€ 38.60)

6 Gerhard Oberhammer (ed.), Beiträge zur Hermeneutik indischer und abendländischer Religionstraditionen. Arbeitsdokumentation eines Symposiums. [SbÖAW 573]. Wien 1991. 256p. (€ 20.30)

7 Helmut Krasser, Dharmottaras kurze Untersuchung der Gültigkeit einer Erkenntnis. Laghuprāmānyapar̄̄sșā (Materialien zur Definition gültiger Erkenntnis in der Tradition Dharmakìrtis 2). Teil 1: Tibetischer Text und Sanskritmaterialien. 162p. Teil 2: Übersetzung. 144p. [SbÖAW 578]. Wien 1991. (€ 25.30)

8 Ernst Steinkellner (ed.), Studies in the Buddhist Epistemological Tradition. Proceedings of the Second International Dharmakīrti Conference, June 11-16, 1989. [DsÖAW 222]. Wien 1991. 430p. (€ 30.50)

9 Gerhard Oberhammer, unter Mitarbeit von Ernst Prets und Joachim Prandstetter, Terminologie der frühen philosophischen Scholastik in Indien. Ein Begriffswörterbuch zur altindischen Dialektik, Erkenntnislehre und Methodologie. Band 1: $A-I$. $[D s \ddot{O A W}$ 223]. Wien 1991. 144p. (€ 18.30)

10 ERIch PILz, Gesellschaftsgeschichte und Theoriebildung in der marxistischen chinesischen Historiographie. Zur Entwicklung der Diskussion um die Han-Gesellschaft. [SbÖAW 582]. Wien 1991. 356p. (€ 40.70) 
11 Susanne Formanek - Sepp Linhart (ed.), Japanese Biographies. Life Histories, Life Cycles, Life Stages. [SbÖAW 590]. Wien 1992. 299p. (€ 25.40)

12 David Jackson, Enlightenment by a Single Means. Tibetan Controversies on the "SelfSufficient White Remedy" (dkar po chig thub). [SbÖAW 615]. Wien 1994. 224p. $(€ 33.80)$

13 Susanne Formanek, Denn dem Alter kann keiner entfliehen. Altern und Alter im Japan der Nara- und Heian-Zeit. [SbÖAW 618]. Wien 1994. 557p. (€ 61.70)

14 Gerhard Oberhammer (ed.), Im Tod gewinnt der Mensch sein Selbst. Das Phänomen des Todes in asiatischer und abendländischer Religionstradition. [SbÖAW 624]. Wien 1995. 356p. (€ 31.60)

15 David Jackson, A History of Tibetan Painting. The Great Tibetan Painters and Their Traditions. [DsÖAW 242]. Wien 1996. 456p., 60 Farbabb., $190 \mathrm{~S} / \mathrm{W}-\mathrm{Abb} ., 2$ Karten. $(€ 101 .-)$

16 Bernhard Scheid, Im Innersten meines Herzens empfinde ich tiefe Scham. Das Alter im Schrifttum des japanischen Mittelalters. [SbÖAW 638]. Wien 1996. 408p. $(€ 52.20)$

17 Gerhard Oberhammer - Ernst Prets - Joachim Prandstetter, Terminologie der frïhen philosophischen Scholastik in Indien. Ein Begriffswörterbuch zur altindischen


1996. 192p. $(€ 30.50)$

18 Friedrich A. Bischoff, Djin Ping Meh. Epitome und analytischer Namenindex gemäß der Übersetzung der Brüder Kibat. [SbÖAW 641]. Wien 1997. 302p. (€ 52.60)

19 Gerhard Oberhammer (ed.), Studies in Hinduism. Vedism and Hinduism. [SbÖAW 642]. Wien 1997. 177p. (€ 24.-)

20 Susanne Formanek - Sepp Linhart (ed.), Aging. Asian Concepts and Experiences. Past and Present. [SbÖAW 643]. Wien 1997. 404p. (€ 63.-)

21 Helmut Krasser - Michael Torsten Much - Ernst Steinkellner - Helmut Tauscher (ed.), Tibetan Studies I \& II (Proceedings of the Yth Seminar of the International Association for Tibetan Studies, Graz 1995, Vol. I-II). [DsÖAW 256]. Wien 1997. 1120p. $(€ 181 .-)$

22 Helmut Eimer (ed.), Transmission of the Tibetan Canon. Papers Presented at a Panel of the 7th Seminar of the International Association for Tibetan Studies, Graz 1995 (PIATS III). [DsÖAW 257]. Wien 1997. 178p. (€ 35.70)

23 Frank J. Korom (ed.), Tibetan Culture in the Diaspora. Papers Presented at a Panel of the 7th Seminar of the International Association for Tibetan Studies, Graz 1995 (PIATS IV) [DsÖAW 258]. Wien 1997. 119p. (€ 30.10)

24 Graham E. Clarke $\dagger$ (ed.), Development, Society, and Environment in Tibet. Papers Presented at a Panel of the 7th Seminar of the International Association for Tibetan Studies, Graz 1995 (PIATS V). [DsÖAW 262]. Wien 1998. 199p., 34 Abb., 20 Tabellen, 4 Karten. (€ 38.-)

25 Deborah E. Klimburg-Salter - Eva Allinger (ed.), The Inner Asian International Style, 12th-14th Centuries. Papers Presented at a Panel of the 7th Seminar of the International Association for Tibetan Studies, Graz 1995 (PIATS VII). [DsÖAW 267]. Wien 1998. 169p. (€ 32.70)

26 Friedrich A. Bischoff, Einführung in die chinesische Schriftsprache. Erster Teil: 
Unterrichtsnotizen zu E. Haenisch, Lehrgang der chinesischen Schriftsprache, Lektionen 1-62. [SbÖAW 660]. Wien 1998. 178p. (€ 24.70)

27 Wolfgang Romanovsky, Die Kriege des Qing-Kaisers Kangxi gegen den Oiratenfürsten Galden. Eine Darstellung der Ereignisse und ihrer Ursachen anhand der Dokumentensammlung "Qing Shilu". [SbÖAW 661]. Wien 1998. 289p. (€ 50.10)

28 Gerhard Oberhammer (ed.), Studies in Hinduism II. Miscellanea to the Phenomenon of Tantras. [SbÖAW 662]. Wien 1998. 103p. (€ 18.80)

29 Siegfried Lienhard, Diamantmeister und Hausväter. Buddhistisches Gemeindeleben in Nepal. [DsÖAW 275]. Wien 1999. 261p., 80 Farbabb. (€ 73.10)

30 Gerhard Oberhammer - Marcus Schmücker (ed.), Raum-zeitliche Vermittlung der Transzendenz. Zur "sakramentalen" Dimension religiöser Tradition. Arbeitsdokumentation eines Symposions. [SbÖAW 665]. Wien 1999. 432p. (€ 57.50)

31 Michael Alram - Deborah E. Klimburg-Salter (ed.), Coins, Art, and Chronology. Essays on the Pre-Islamic History of the Indo-Iranian Borderlands. [DsÖAW 280]. Wien 1999. 498p., 123 Abb., 35 Tafeln, 8 Karten. (vergriffen)

32 Shoryu Katsura (ed.), Dharmakirti's Thought and Its Impact on Indian and Tibetan Philosophy. Proceedings of the Third International Dharmakīrti Conference, Hiroshima, November 4-6, 1997. [DsÖAW 281]. Wien 1999. 474p., 1 Abb. (€ 71.90)

33 Marion Rastelli, Philosophisch-theologische Grundanschauungen der Jayākhyasamhitā. Mit einer Darstellung des täglichen Rituals. [SbÖAW 668]. Wien 1999. 433p. (€ 64.60)

34 Мотог Ono, Prajñākaraguptas Erklärung der Definition gültiger Erkenntnis (Pramānavārttikālamkāara zu Pramānavārttika II 1-y). Teil I: Sanskrit-Text und Materialien. [SbÖAW 678]. Wien 2000. 108p. (€ 28.70)

35 H. Brunner - G. Oberhammer - A. Padoux (ed.), Tāntrikābhidhānakośa I. Dictionnaire des termes techniques de la littérature hindoue tantrique. A Dictionary of Technical Terms from Hindu Tantric Literature. Wörterbuch zur Terminologie hinduistischer Tantren. [SbÖAW 681]. Wien 2000. 260p. (€ 41.90)

36 T. Gyalbo - G. Hazod - P.K. Sörensen, Civilisation at the Foot of Mount Sham-po. The Royal House of $l H a$ Bug-pa-can and the History of g.Ya'-bzang. Historical Texts from the Monastery of g. Ya'-bzang in Yar-stod (Central Tibet). Annotated Translation, Transliteration and Facsimile Edition. [DsÖAW 290]. Wien 2000. 340p., 1 Faltplan. $(€ 67.80)$

37 P. Wangdu - H. Diemberger, dBa'bzhed. The Royal Narrative Concerning the Bringing of the Buddhas Doctrine to Tibet. Translation and Facsimile Edition of the Tibetan Text. [DsÖAW 291]. Wien 2000. 121 p. (€ 28.40)

38 Bernhard Scheid, Der eine und einzige Weg der Götter. Yoshida Kanemoto und die Erfindung des Shinto. [SbÖAW 687]. Wien 2001. 393 p. (€ 56.10)

39 Helmut Krasser, Śañkarānandanas İśvarāpākaranasañkṣepa mit einem anonymen Kommentar und weiteren Materialien zur buddhistischen Gottespolemik. Teil 1: Texte. xxii + 127p. Teil 2: Annotierte Übersetzungen und Studie zur Auseinandersetzung über die Existenz Gottes. 378p. [SbÖAW 689]. Wien 2002 (€ 72.-)

40 Gerhard Oberhammer - Marion Rastelli (ed.), Studies in Hinduism III. Pāñcarātra and Viśiștādvaitavedānta. [SbÖAW 694]. Wien 2002. 152p. (€ 36.50)

41 Gerhard Oberhammer - Marcus Schmücker (ed.), Mythisierung der Transzendenz als Entwurf ihrer Erfahrung. Arbeitsdokumentation eines Symposiums. [SbÖAW 706]. Wien 2003. 419p. (€ 59.20) 
42 Susanne Formanek - William Lafleur (ed.), Practicing the Afterlife. Perspectives from Japan. [SbÖOW 713]. Wien 2004. 536p. (€ 69.80)

43 Eli Franco, The Spitzer Manuscript. The Oldest Philosophical Manuscript in Sanskrit. Vol. I-II. [DsÖAW 323]. Wien 2004. xii + 510p. (€ 139.-)

44 H. Brunner - G. Oberhammer - A. Padoux (ed.), Tāntrikābhidhānakośa II. Dictionnaire des termes techniques de la littérature hindoue tantrique. A Dictionary of Technical Terms from Hindu Tantric Literature. Wörterbuch zur Terminologie hinduistischer Tantren. [SbÖAW 714]. Wien 2004. 308p. (€ 55.40)

45 Friedrich Bischoff, San tzu ching Explicated - The Classical Initiation to Classic Chinese Couplet I to XI. [SbÖAW 719]. Wien 2005. 310p. (€ 52.-)

46 Per Kueld Sørensen - Guntram Hazod, Thundering Falcon. An Inquiry into the History and Cult of Khra-'brug, Tibet's First Buddhist Temple. [DsÖAW 333]. Wien 2005. 432p. (€99.36)

47 Susanne Formanek, Die „böse Alte“ in der japanischen Populärkultur der Edo-Zeit. Die Feindvalenz und ihr soziales Umfeld. [SbÖAW 727]. Wien 2005. 566p. (€ 66.40)

48 Hartmut Walravens (ed.), Joseph Franz Rock: Briefwechsel mit E.H. Walker 19381961. [SbÖAW 738]. Wien 2006. 328p. (€ 49.-)

49 Gerhard Oberhammer - Ernst Prets - Joachim Prandstetter, Terminologie der frühen philosophischen Scholastik in Indien. Ein Begriffswörterbuch zur altindischen Dialektik, Erkenntnislehre und Methodologie. Band 3: Pra-H. [DsÖAW 343]. Wien 2006. 305p. (€ 94.80)

50 Susanne Weigelin-Schwiedrzik - Agnes Schick-Chen - Sascha Klotzbücher (ed.), As China Meets the World. China's Changing Position in the International Community. [SbÖAW 747]. Wien 2006. 210p. (€ 31.20)

51 Marion Rastelli, Die Tradition des Pāñcarātra im Spiegel der Parameśvarasamhitā. [SbÖAW 748]. Wien 2006. 614p. (€ 55.20)

52 Cathy Cantwell - Robert Mayer, The Kīlaya Nirvāna Tantra and the Vajra Wrath Tantra. Two Texts from the Ancient Tantra Collection. [DsÖAW 349]. Wien 2007. 298p. $(€ 65.20)$

53 Karin Preisendanz (ed.), Expanding and Merging Horizons. Contributions to South Asian and Cross-Cultural Studies in Commemoration of Wilhelm Halbfass. [DsÖAW 351]. Wien 2007. xv + 732p. (€95.20)

54 Gerhard Oberhammer - Marion Rasteldi (ed.), Studies in Hinduism IV. On the Mutual Influences and Relationship of Viśiștādvaita Vedānta and Pāñcarātra. [SbÖAW 756]. Wien 2007. 325p. (€60.-)

55 Hartmut Walravens, Joseph Franz Rock (1884-1962): Tagebuch der Reise von Chieng Mai nach Yünnan, 1921-1922. Briefwechsel mit C. S. Sargent, University of Washington, Johannes Schubert und Robert Koc. [SbÖAW 757]. Wien 2007. 580p. (€ 69.60)

56 Vincent Eltschinger, Penser l'autorité des Écritures. La polémique de Dharmakīrti contre la notion brahmanique orthodoxe d'un Veda sans auteur. Autour de Pramānavārttika 1.213-268 et Svavṛtti. [SbÖAW 760]. Wien 2007. 651p. (€ 55.20)

57 Hartmut Buescher, Sthiramati's Trimssikāvijñaptibhāsya. Critical Editions of the Sanskrit Text and its Tibetan Translation. [SbÖAW 768]. Wien 2007. 157p. (€ 31.20)

58 David Seyfort Ruegg, The Symbiosis of Buddhism with Brahmanism/Hinduism in South Asia and of Buddhism with "Local Cults" in Tibet and the Himalayan Region. [SbÖAW 774]. Wien 2008. 197p. (€ 35.50) 
59 Adelheid Herrmann-Pfandt, Die lHan kar ma. Ein früher Katalog der ins Tibetische übersetzten buddhistischen Texte. Kritische Neuausgabe mit Einleitung und Materialien. [Ds $\ddot{O} A W 367]$. Wien 2008. 531p. (€ 96.-)

60 Gerhard Oberhammer - Marcus Schmücker (ed.), Glaubensgewissheit und Wahrheit in religiöser Tradition. Arbeitsdokumentation eines Symposiums. [SbÖAW 775]. Wien 2008. 349p. (€ 52.60)

61 John Vincent Bellezza, Zhang Zhung. Foundations of Civilization in Tibet. A Historical and Ethnoarchaeological Study of the Monuments, Rock Art, Texts, and Oral Tradition of the Ancient Tibetan Upland. [DsÖAW 368]. Wien 2008. 841p. (€ 129.20)

62 Hartmut Buescher, The Inception of Yogācāra-Vijñānavāda. [SbÖAW 776]. Wien 2008. 229p. (€ 49.-)

63 Cathy Cantwell - Robert Mayer, Early Tibetan Documents on Phur pa from Dunhuang. [DsÖAW 370]. Wien 2008. 228p. (€ 66.-)

64 Manfred Taube (ed.), Briefwechsel J.F. Rock - J. Schubert 1935-1961. [SbÖAW 782]. Wien 2009. 315p. (€ 37.90)

65 Eli Franco in collaboration with Dagmar Eigner (ed.), Yogic Perception, Meditation and Altered States of Consciousness. [SbÖAW 794]. Wien 2009. vii + 483p. (€ 42.-)

66 Johannes SchneIder, Vāgīsvarakīrtis Mṛtyuvañcanopadeśa, eine buddhistische Lehrschrift zur Abwehr des Todes. [DsÖAW 394]. Wien 2010. vi + 299p. (€ 74.-)

67 Hartmut Walravens (ed.), Josef Franz Rock. Phytogeography of Northwest and Southwest China. Edited with indices. [SbÖAW 799]. Wien 2010. 356p. (€ 59.20)

68 Kei Kataoka, Kumārila on Truth, Omniscience and Killing. Part 1: A Critical Edition of Mīmāmsā-Ślokavārttika ad 1.1.2 (Codanāsūtra). Part 2: An Annotated Translation of Mīmāṃsā-Ślokavārttika ad 1.1.2 (Codanāsūtra). [SbÖAW 814]. Wien 2011. $97+627$ p. (€ 85.-)

69 Helmut Krasser - Horst Lasic - Eli Franco - Birgit Kellner (ed.), Religion and Logic in Buddhist Philosophical Analysis. Proceedings of the Fourth International Dharmakīrti Conference, Vienna, August 23-27, 2005. [DsÖAW 424]. Wien 2011. 521p. $(€ 69 .-)$

70 Gerhard Oberhammer - Marcus Schmücker (ed.), Die Relationalität des Subjektes im Kontext der Religionshermeneutik. [SbÖAW 816]. Wien 2011. 351p. (€ 48.60)

71 Hartmut Walravens (ed.), Nikolaus Poppe (189\%-1991). Briefwechsel mit Walther Heissig (1913-2005). Ein Beitrag zur Geschichte der Mongolistik. [SbÖAW 817]. Wien 2011. 326p. (€ 49.-)

72 Max Deeg - Oliver Freiberger - Christoph Kleine (ed.), Kanonisierung und Kanonbildung in der asiatischen Religionsgeschichte. [SbÖOW 820]. Wien 2011. 536p. (€ 79.52)

Materialien zur Kultur- und Geistesgeschichte Asiens

1 Susanne Formanek - Peter Getreuer, Verzeichnis des deutschsprachigen JapanSchriftums 1980-198\%. Wien 1989. 194p. (€ 15.20)

2 Peter Getreuer, Verzeichnis des deutschsprachigen Japan-Schrifttums 1988-1989. Nebst Ergänzungen zu den Jahren 1980-1987. Wien 1991. 181p. (€ 15.20) 
3 Karina Kleiber, Verzeichnis des deutschsprachigen Japan-Schriftums 1990-1991. Nebst Ergänzungen zu den Jahren 1980-1989. Wien 1995. 194p. (€ 31.90)

4 Gabriele Pauer, Verzeichnis des deutschsprachigen Japan-Schrifttums 1992-1993. Nebst Ergänzungen zu den Jahren 1980-1991. Wien 1999. 228p. (€ 47.20)

5 Karina Kleiber, Verzeichnis des deutschsprachigen Japan-Schriftums 1994-199\%. Nebst Ergänzungen zu den Jahren 1980-1993. Wien 2002. 463p. (€ 57.-)

Sanskrit Texts from the Tibetan Autonomous Region

1 Ernst Steinkellner - Helmut Krasser - Horst Lasic (ed.), Jinendrabuddhi's Viśálāmalavat̄̄ Pramānasamuccayațīkā. Chapter 1. Part I: Critical Edition-Part II: Diplomatic Edition with a Manuscript Description by Anne MacDonald. Beijing: China Tibetology Publishing House - Vienna: Austrian Academy of Sciences Press, 2005. lx +209 p. \& xl + 161p. (€ 39.-)

2 Ernst Steinkellner (ed.), Dharmakīti's Pramānaviniścaya, Chapters 1 and 2. Beijing: China Tibetology Publishing House - Vienna: Austrian Academy of Sciences Press, 2007. 142p. (€ 31.20)

4 Xuezhu Li - Ernst Steinkellner (ed.), Vasubandhu's Pañcaskandhaka. Beijing: China Tibetology Publishing House - Vienna: Austrian Academy of Sciences Press, 2008. 107p. (€ 28.-)

5 Ernst Steinkellner - Toru Tomabechi (ed.), The Adhyardhaśatikā Prajñapāramitā. Sanskrit and Tibetan Texts. Beijing: China Tibetology Publishing House - Vienna: Austrian Academy of Sciences Press, 2009. xlviii + 100p. (€ 28.-)

6 Ernst Steinkellner - Luo Hong - Toru Tomabechi (ed.), Candrakīrti's Vajrasattvanispādanasūtra (Vajrasattvasādhana). Sanskrit and Tibetan Texts. Beijing: China Tibetology Publishing House - Vienna: Austrian Academy of Sciences Press, 2009. 100p. $(€ 29.50)$

7 Bhikṣuñī Vinītā (ed.), A Unique Collection of Twenty Sütras in a Sanskrit Manuscript from the Potala. 2 vols. Beijing: China Tibetology Publishing House - Vienna: Austrian Academy of Sciences Press, 2010. 846p. (€ 118.-)

8 Pascale Hugon - Toru Tomabechi (ed.), Dharmakīrti's Pramānaviniścaya, Chapter 3. Beijing: China Tibetology Publishing House - Vienna: Austrian Academy of Sciences Press, 2012. 223p. (€ 45.-)

Wiener Zeitschrift für die Kunde Südasiens

(Vienna Journal of South Asian Studies)

Band XXXVIII, 1994. Roque Mesquita - Chlodwig. H. Werba (ed.), Orbis Indicus. Festschrift für Gerhard Oberhammer zum 65. Geburtstag. 523p. (€ 71.20)

Band XXXIX, 1995. 282p. (€ 43.40)

Band XL, 1996. 242p. (€ 41.90)

Band XLI, 1997. 251p. (€ 45.40)

Band XLII, 1998. 240p. (€ 46.90)

Band XLIII, 1999. 264p. (€ 50.-) 
Band XLIV, 2000. 255p. (€ 50.50)

Band XLV, 2001. 248p. (€ 51.20)

Band XLVI, 2002. 310p. (€ 69.-)

Band XLVII, 2003. 264p. (€ 56.-)

Band XLVIII, 2004. 262p. (€ 66.-)

Band XLIX, 2005. 283p. (€ 69.-)

Band L, 2006. 245p. (€ 48.40)

Band LI, 2007-2008. 262p. (€ 48.40)

Band LII-LIII, 2009-2010 (2010). 358p. (€ 58.-)

Bestellungen direkt beim Verlag:

Verlag der Österreichischen Akademie der Wissenschaften

Postfach 471, Postgasse 7/4

A-1011 Wien

Tel.: 0043-1-51581/3401-3406

Fax: 0043-1-51581/3400

E-mail:verlag@oeaw.ac.at

Homepage: http://verlag.oeaw.ac.at

Für die noch verfügbaren Bände VI, 1962 - XI, 1967 und XV, 1971 - XXXVII, 1993 richten Sie Ihre Bestellung bitte an:

Institut für Südasien-, Tibet- und Buddhismuskunde - Bereich Südasienkunde Campus der Universität Wien

Spitalgasse 2, Hof 2/2.1

A-1090 Wien

Tel.: 0043-1-4277-43501

Fax: 0043-1-4277-9435

E-mail: istb@univie.ac.at

Homepage: http://www.istb.univie.ac.at 
